\title{
Special issue of The Journal of Antibiotics dedicated to the late Professor C Richard Hutchinson
}

\author{
The Journal of Antibiotics (2011) 64, 3-5; doi:10.1038/ja.2010.158
}

$\mathrm{I}^{\mathrm{t}}$

$\mathrm{t}$ is our distinct privilege as Guest Editors of this special issue of The Journal of Antibiotics to honor the late Professor C Richard Hutchinson with a diverse collection of Reviews, Original Research Articles and Notes on various aspects of natural product discovery, biosynthesis, engineering, and drug discovery and development, passionately contributed by many of his former students, postdoctoral fellows, collaborators, colleagues and friends. These contributions serve as a reminder of the remarkable impact that extraordinarily passionate individual can have on any chosen discipline.

C Richard 'Dick'/'Hutch' Hutchinson, PhD, Edward Leete Professor Emeritus of Medicinal Chemistry and Professor Emeritus of Bacteriology, University of Wisconsin-Madison, died on 5 January 2010 at the age of 66 after a courageous 3-month fight against cancer. ${ }^{1}$ During a distinguished career that spanned nearly four decades at the University of Connecticut, University of Wisconsin-Madison, Kosan Biosciences and Centrose LLC, Professor Hutchinson trained many future academicians and industrial leaders, touched many lives with his wisdom, made major advances in understanding the biosynthesis of naturally occurring drugs through studies of the molecular genetics and biochemistry of antibiotics production in microorganisms, and worked tirelessly and enthusiastically through creative entrepreneurship to translate laboratory findings into clinical products.

Professor Hutchinson's early training and independent work were in chemistry, where he made many important contributions to alkaloid biosynthesis. He initially focused on the biosynthesis of plant products such as camptothecin and iridoids. Even to this day his studies on camptothecin represent most of what we know about the biosynthesis of this important clinical compound. ${ }^{2-5} \mathrm{He}$ extended his work into microbial natural products, beginning with macrolide brefeldin $A^{6-11}$ and polyether lasalocid $A^{12-16}$ in the late 1970s. In the early 1980s, attracted to microbial genetics, he gradually and, eventually, entirely shifted the focus of his biosynthetic work toward a genetic and biochemical approach. For the following 20 years, he studied the biosynthesis of many biomedically important polyketide natural products, heavily pioneering the field of combinatorial biosynthesis and natural product drug discovery. Professor Hutchinson was one of the world leaders who elegantly blended the art and science of chemistry, biochemistry and molecular biology to understand secondary metabolite biosynthesis in actinomycetes, and was one of the very first chemists to master and combine these fields.

Professor Hutchinson made seminal contributions to both type I and type II polyketide synthases (PKSs). For type II PKSs, he is best known for his work on the tetracenomycin $(\mathrm{Tcm})$ and daunorubicin

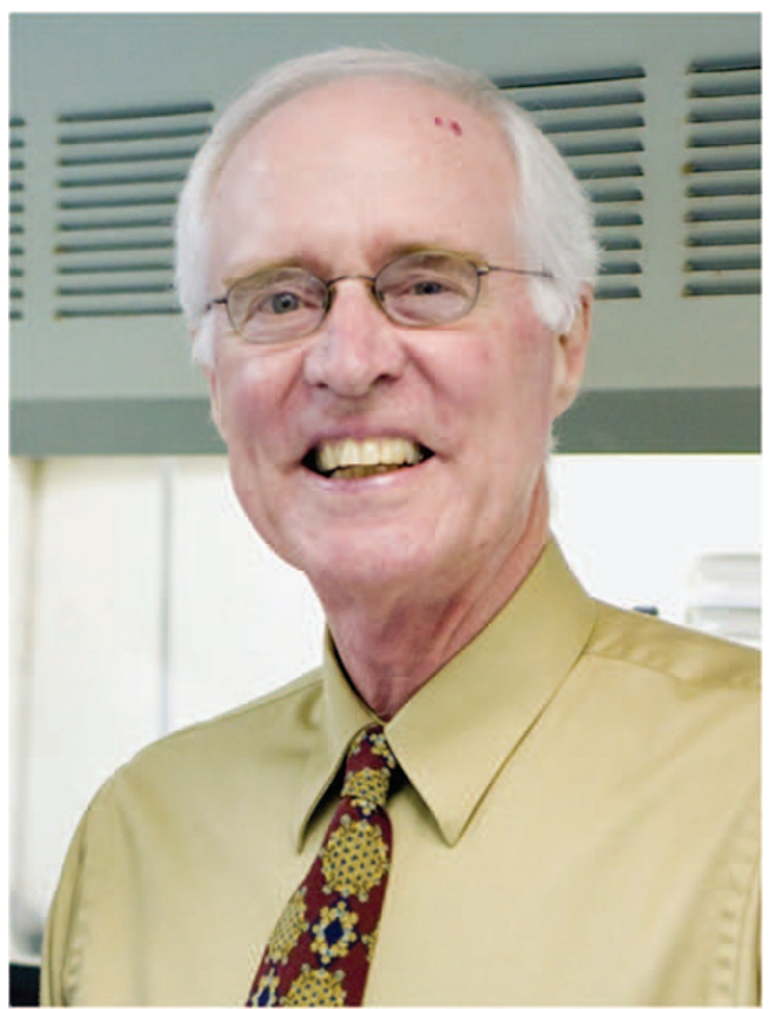

(Dnr) biosynthesis gene clusters. ${ }^{17}$ Legacies of his work include (i) the tcm and $d n r$ clusters as two of the best-characterized type II PKS gene clusters to date, serving as models for aromatic polyketide biosynthesis in general; ${ }^{18-21}$ (ii) in vitro reconstitution and characterization of the Tcm PKS, which opened the possibility to study the enzyme mechanism and structure of type II PKS in vitro; ${ }^{22}$ and (iii) cross-talk between fatty acid biosynthesis and $\mathrm{Tcm}$ biosynthesis in Streptomyces glaucescens, which led to the identification and subsequent confirmation of the malonyl CoA:acyl carrier protein transferase as the missing link between fatty acid (primary metabolism) and polyketide biosynthesis (secondary metabolism). ${ }^{23}$ For type I PKSs, he is best known for his work on erythromycin, tylosin and rifamycin (Rif) biosynthesis gene clusters. Highlights from his work in this field include (i) his hypothesis and demonstration by feeding experiments that type I PKS acts by a processive mechanism, which was widely considered as a milestone for macrolide biosynthesis; this conceptual advance was 
critical for the subsequent discovery and characterization of the modular type I PKS; ${ }^{24}$ (ii) the fundamental studies on erythromycin biosynthesis in Saccharopolyspora erythraea, which were critical to the eventual discovery of the very first modular type I PKS; ${ }^{25-30}$ and (iii) characterization of the rif cluster and isolation of a family of elongating polyketide intermediates, which experimentally confirmed for the first time the processive mechanism of type I PKS. ${ }^{31-33}$

Professor Hutchinson was a pioneer of combinatorial biosynthesis for the structural diversity of natural products. The most notable contribution of his laboratory was the development of methodologies, strategies and concepts dictating effective combinatorial biosynthesis. Highlights from his laboratory include (i) the demonstration that the ketoacyl synthase $\alpha$ - and $\beta$-subunits of type II PKS both contribute to the chain length for aromatic polyketide biosynthesis; ${ }^{34,35}$ (ii) the recognition that synthesis of aromatic polyketides bearing starter units other than acetate requires dedicated enzymes that interact with the type II PKS complex; ${ }^{36,37}$ (iii) exploration of environmental DNAs for the production of complex natural products; ${ }^{38}$ and (iv) the production of epirubicin, a commercially important clinical drug, by a metabolically engineered organism, ${ }^{39}$ the only example known to date that has been practically advanced by combinatorial biosynthesis.

While his early work on brefeldin A still represents some of the best examples of fungal polyketide biosynthesis by the traditional approaches, ${ }^{6-11}$ Professor Hutchinson returned to fungal polyketides in the 1990s, but adopting a more contemporary approach. Recognizing that fungal PKSs are mechanistically and structurally distinct from bacterial PKSs and that fungal polyketides represent a major source of clinically important natural products, he worked on lovastatin biosynthesis in Aspergillus terreus as a model system for polyketide biosynthesis in fungi. The cloning, sequencing and characterization of lovastatin gene cluster unveiled a novel type of PKS and set the stage for the engineering and production of novel lovastatin analogs, ${ }^{40,41}$ a class of the most successful antihypercholesterolemic drugs on the market.

Professor Hutchinson helped to create the mold for what is now termed 'translational research'. He was integral to the operations of numerous pharmaceutical companies. After retiring from the University of Wisconsin-Madison in 2000, he joined Kosan Biosciences, Hayward, CA as a Vice President of New Technologies. He returned part-time to the University of Wisconsin-Madison in 2004 to help run the University of Wisconsin-Madison National Cooperative Drug Discovery Group. In December 2006 he left Kosan and shortly thereafter co-founded Centrose LLC, Madison, WI. Most recently, he served as the President and Chief Scientific Officer of Centrose LLC.

Professor Hutchinson was an extraordinary teacher and mentor who trained and inspired a generation of young scientists in the chemistry, biochemistry and molecular biology of secondary metabolite biosynthesis. During his tenure at the University of Connecticut (1971-1974) and University of Wisconsin-Madison (1977-2000), Professor Hutchinson trained and mentored over 100 graduate students, postdoctoral fellows and visiting scientists. Many of these people are now successful scientists at both academic and industrial institutions nationally and internationally. A symposium, titled Natural Product Biosynthesis, Engineering, and Drug Discovery, honoring Professor Hutchinson's distinguished career and featuring 11 presentations by former Hutch Lab members, on the occasion of his 65th birthday, was held in 2008. The symposium was attended by more than 150 former lab members, collaborators, colleagues and friends. All were, and continue to be, grateful beyond words to have had the opportunity to celebrate Professor Hutchinson's accomplishments and life example and to show their appreciation to his tremendous contributions to his beloved profession. The annual C Richard
Hutchinson Lecture was established in 2008 at the School of Pharmacy, University of Wisconsin-Madison, ensuring that Professor Hutchinson's legacy of scientific rigor, progressivism and standard of excellence will be continued.

Finally, we would like to express our sincere gratitude and appreciation to the authors for their outstanding contributions to this special issue dedicated to Professor Hutchinson. One of us (BS) also would like to take this opportunity to pay his tribute to Professor Hutchinson for the scholarly and scientific advice and mentorship that has so profoundly impacted his career, as well as the friendship, for which he and his family are forever grateful. Professor Hutchinson will be remembered for his strength of spirit, zeal for life and new experiences, and his boundless intellectual curiosity. He has left an indelible mark, not only in our field of science, but even more so on the lives of those lucky enough to have learned from him and who considered him a friend and a role model.

\section{Ben Shen ${ }^{1}$ and Haruo Ikeda ${ }^{2}$} Guest Editors

${ }^{1}$ Division of Pharmaceutical Sciences, School of Pharmacy \& Department of Chemistry, University of Wisconsin-Madison, Madison, WI, USA and ${ }^{2}$ Laboratory of Microbial Engineering, Kitasato Institute for Life Sciences, Kitasato University, Minami-ku, Sagamihara, Kanagawa, Japan E-mail: bshen@pharmacy.wisc.edu or ikeda@ls.kitasato-u.ac.jp

1 Shen, B. Obituary: Professor Emeritus C Richard Hutchinson. J. Antibiot. 63, 409-410 (2010).

2 Hutchinson, C. R., Heckendorf, A. H., Daddona, P. E., Hagaman, E. \& Wenkert, E. Biosynthesis of camptothecin. I. Definition of the overall pathway assisted by carbon-13 nuclear magnetic resonance analysis. J. Am. Chem. Soc. 96, 5609-5611 (1974).

3 Heckendorf, A. H. \& Hutchinson, C. R. Biosynthesis of camptothecin. II. Confirmation that isovincoside, not vincoside, is the penultimate biosynthetic precursor of indole alkaloids. Tetrahedron Lett. 18, 4153-4154 (1977).

4 Hutchinson, C. R., Heckendorf, A. H., Straughn, J. L., Daddona, P. E. \& Cane, D. E. Biosynthesis of camptothecin. III. Definition of strictosamide as the penultimate biosynthetic precursor assisted by ${ }^{13} \mathrm{C}$ and ${ }^{2} \mathrm{H}$ NMR spectroscopy. J. Am. Chem. Soc. 101, 3358-3369 (1979).

5 Hutchinson, C. R. Camptothecin: chemistry, biogenesis, and medicinal chemistry. Tetrahedron 37, 1047-1065 (1981).

6 Mabuni, C. T., Garlaschelli, L., Ellison, R. A. \& Hutchinson, C. R. Biosynthetic origin of the oxygen atoms in the $C_{15}$ macrolide antibiotic brefeldin A. J. Am. Chem. Soc. 99, 7718-7720 (1977).

7 Mabuni, C. T., Garlaschelli, L., Ellison, R. A. \& Hutchinson, C. R. Biosynthesis of the $\mathrm{C}_{15}$ macrolide antibiotics. 1. Biochemical origin of the four oxygen atoms in brefeldin $A$. J. Am. Chem. Soc. 101, 707-714 (1979).

8 Hutchinson, C. R. et al. Biosynthesis of macrolide antibiotics. 3. Regiochemistry of isotopic hydrogen labeling of brefeldin A by acetate. J. Am. Chem. Soc. 103, 24742477 (1981).

9 Hutchinson, C. R., Kurobane, I., Cane, D. E., Hasler, H. \& McInnes, A. G. Biosynthesis of macrolide antibiotics. 4. Stereochemistry of hydrogen labeling of brefeldin A by [2- ${ }^{2} \mathrm{H}_{3}$ ]acetate. J. Am. Chem. Soc. 103, 2477-2480 (1981).

10 Yamamoto, Y., Hori, A. \& Hutchinson, C. R. Biosynthesis of macrolide antibiotics. 6. Late steps in brefeldin A biosynthesis. J. Am. Chem. Soc. 107, 2471-2474 (1985).

11 Gonzalez de la Parra, M. \& Hutchinson, C. R. Nonstereospecific hydrogen exchange in the biosynthesis of the macrolide antibiotic, brefeldin A. J. Am. Chem. Soc. 108, 2448-2449 (1986).

12 Hutchinson, C. R., Sherman, M. M., Mclnnes, A. G., Walter, J. A. \& Vederas, J. C. Biosynthesis of macrolides. 6 . Mechanism of stereocontrol during the formation of Lasalocid A. J. Am. Chem. Soc. 103, 5956-5959 (1981).

13 Hutchinson, C. R., Sherman, M. M., Vederas, J. C. \& Nakashima, T. T. Biosynthesis of macrolides. 5. Regiochemistry of the labeling of Lasalocid $A$ by $\left[{ }^{13} \mathrm{C},{ }^{18} \mathrm{O}\right]$-labeled precursors. J. Am. Chem. Soc. 103, 5953-5956 (1981).

14 Sherman, M. M., Yue, S. \& Hutchinson, C. R. Biosynthesis of Lasalocid A. Metabolic interrelationships of carboxylic acid precursors and polyether antibiotics. J. Antibiot. 39, 1135-1143 (1986).

15 Sherman, M. M. \& Hutchinson, C. R. Biosynthesis of Lasalocid A: biochemical mechanism for assembly of the carbon framework. Biochemistry 26, 438-445 (1977).

16 Sherman, M. M. \& Hutchinson, C. R. Biosynthesis of Lasalocid A. Biochemical alteration of polyether antibiotic production. J. Antibiot. 39, 1270-1280 (1986).

17 Hutchinson, C. R. Biosynthetic studies of daunorubicin and tetracenomycin C. Chem. Rev. 97, 2525-2535 (1997). 
18 Malpartida, F. et al. Homology between Streptomyces genes coding for synthesis of different polyketides used to clone antibiotic biosynthetic genes. Nature $\mathbf{3 2 5}$, 818-821 (1987).

19 Motamedi, H. \& Hutchinson, C. R. Cloning and heterologous expression of a gene cluster for the biosynthesis of tetracenomycin C, the anthracycline antitumor antibiotic of Streptomyces glaucescens. Proc. Natl Acad. Sci. USA 84, 4445-4449 (1987).

20 Bibb, M. J., Biro, S., Motamedi, H., Collins, J. F. \& Hutchinson, C. R. Analysis of the nucleotide sequence of the Streptomyces glaucescens tcml genes provides key information about the enzymology of polyketide antibiotic biosynthesis. EMBO J. 8, 2727-2736 (1989)

21 Stutzman-Engwall, K. J. \& Hutchinson, C. R. Multigene families for anthracycline antibiotic production in Streptomyces peucetius. Proc. Natl Acad. Sci. USA 86, !3135-3139 (1989)

22 Shen, B. \& Hutchinson, C. R. Enzymatic synthesis of a bacterial polyketide from acetyl and malonyl coenzyme A. Science 262, 1535-1540 (1993).

23 Summers, R. G., Ali, A., Shen, B., Wessel, W. A. \& Hutchinson, C. R. Malonylcoenzyme A:acyl carrier protein acyltransferase of Streptomyces glaucescens: a possible link between fatty acid and polyketide biosynthesis. Biochemistry 34, 9389-9402 (1995).

24 Yue, S., Duncan, J. S., Yamamoto, Y. \& Hutchinson, C. R. Macrolide biosynthesis Tylactone formation involves the processive addition of three carbon units. J. Am. Chem. Soc. 109, 1253-1255 (1987).

25 Wang, Y. G., Davies, J. E. \& Hutchinson, C. R. Plasmid DNA in the erythromycin producing microorganism, Streptomyces erythreus NRRL 2338. J. Antibiot. 35, 335342 (1982).

26 Weber, J. M., Wierman, C. K. \& Hutchinson, C. R. Genetic analysis of erythromycin production in Streptomyces erythreus. J. Bacteriol. 164, 425-433 (1985).

27 Yamamoto, H., Maurer, K. H. \& Hutchinson, C. R. Transformation of Streptomyces erythraeus. J. Antibiot. 39, 1304-1313 (1986).

28 Shafiee, A. \& Hutchinson, C. R. Macrolide antibiotic biosynthesis: isolation and properties of two forms of 6-deoxyerythronolide B hydroxylase from Saccharopolyspora erythraea (Streptomyces erythreus). Biochemistry 26, 6204-6210 (1987).

29 Donadio, S. \& Hutchinson, C. R. Cloning and characterization of the Saccharopolyspora erythraea $f d x A$ gene encoding Ferredoxin. Gene 100, 231-235 (1991).
30 Andersen, J. F. \& Hutchinson, C. R. Characterization of Saccharopolyspora erythraea cytochrome P-450 genes and enzymes, including 6-deoxyerythronolide B hydroxylase. J. Bacteriol. 174, 725-735 (1992).

31 August, P. R. et al. Biosynthesis of the ansamycin antibiotic Rifamycin: deductions from the molecular analysis of the rif biosynthetic gene cluster of Amycolatopsis mediterranei S699. Chem. Biol. 5, 69-79 (1998).

$32 \mathrm{Yu}, \mathrm{T}$. W. et al. Direct evidence that the rifamycin polyketide synthase assembles polyketide chains processively. Proc. Natl Acad. Sci. USA 96, 9051-9056 (1999).

33 Doi-Katayama, Y. et al. Thioesterases and the premature termination of polyketide chain elongation in rifamycin B biosynthesis by Amycolatopsis mediterranei S699. J. Antibiot. 53, 484-495 (2000).

34 Shen, B., Summers, R. G., Wendt-Pienkowski, E. \& Hutchinson, C. R. The Streptomyces glaucescens $t c m K L$ polyketide synthase and $t c m N$ polyketide cyclase genes govern the size and shape of aromatic polyketides. J. Am. Chem. Soc. 117, 6811-6821 (1995).

35 Shen, B. \& Hutchinson, C. R. Deciphering the mechanism for the assembly of aromatic polyketides by a bacterial polyketide synthase. Proc. Natl Acad. Sci. USA 93, 6600-6604 (1996).

36 Bao, W., Sheldon, P. J. \& Hutchinson, C. R. Purification and properties of the Streptomyces peucetius DpsC -ketoacyl:acyl carrier protein synthase III that specifies the propionate-starter unit for type II polyketide biosynthesis. Biochemistry 38, 9752-9757 (1999).

37 Bao, W., Sheldon, P. J., Wendt-Pienkowski, E. \& Hutchinson, C. R. The Streptomyces peucetius dpsC gene determines the choice of starter unit in biosynthesis of the daunorubicin polyketide. J. Bacteriol. 181, 4690-4695 (1999).

38 Seow, K. T. et al. A study of iterative type II polyketide synthases, using bacterial genes cloned from soil DNA: a means to access and use genes from uncultured microorganisms. J. Bacteriol. 179, 7360-7368 (1997).

39 Madduri, K. et al. Production of the antitumor drug Epirubicin (4'-epidoxorubicin) and its precursor by a genetically engineered strain of Streptomyces peucetius. Nat. Biotechnol. 16, 69-74 (1998).

40 Kennedy, J. et al. Modulation of polyketide synthase activity by accessory proteins during lovastatin biosynthesis. Science 284, 1368-1372 (1999).

41 Auclair, K. et al. Lovastatin nonaketide synthase catalyzes an intramolecular DielsAlder reaction of a substrate analogue. J. Am. Chem. Soc. 122, 11519-11520 (2000). 